\title{
PREVALENCIA DEL ALCOHOLISMO DURANTE TRES DÉCADAS EN CHILE (1952-1982)
}

Pedro Naveillan*

Sergio Vargas*

\begin{abstract}
NAVEILLAN, P. \& VARGAS, S. Prevalencia del alcoholismo durante tres décadas en Chile (19521982). Rev. Saúde públ., S. Paulo, 23:128-35, 1989.

RESUMEN: Mediante el método de Jellinek se estudió la evolución del número de alcohólicos y de la tasa de prevalencia de alcoholismo en Chile y sus variaciones entre los años 1952 y 1982 . Se señalan los sesgos y limitaciones de la metodología empleada y se comparan los resultados obtenidos con los de otras investigaciones chilenas. Se concluye que ha habido un incremento del número de alcohólicos y de la tasa de alcoholismo en el periodo analizado que para 1982 es un $70 \%$ mayor que para 1952. Este incremento ha sido irregular y fluctuante. Nuestros resultados cuestionan la eficacia de las políticas de prevención del alcoholismo llevadas a cabo en el período.
\end{abstract}

DESCRIPTORES: Alcoholismo, incidencia. Prevalencia.

\section{INTRODUCCIÓN}

Nos ha parecido de interés formarnos una idea en un país como Chile - poseedor de una larga trayectoria en su conocimiento teórico e investigación básica y aplicada y su visualización como problema de salud pública - de la evolución en el tiempo de la prevalencia del alcoholismo.

El pleno reconocimiento en nuestro país del alcoholismo como enfermedad comienza a lograrse en la década de los años 1950 y recibe su consagración definitiva hacia 1957, con las ideas y visita de E.M. Jellinek.

Un grupo importante de profesionales de la salud, mayoritariamente del área psiquiátrica, ya se había interesado por esta problemática y realizado algunos estudios epidemiológicos de campo $2,3,4,6,12,13$. La presencia de Jellinek permitio una mutua fertilización que se tradujo en numerosos trabajos de investigación y en programas de prevención primaria, secundaria y terciaria del alcoholismo. Lo anteriormente señalado condujo a una toma de conciencia, que nos atreveríamos a llamar nacional, acerca de la magnitud y gravedad de esta problemática, claramente de salud pública y merecedora de una prioridad más urgente que la que recibido hasta la actualidad.

En las más de tres décadas transcurridas desde los años cincuenta a la fecha, se han multiplicado los servicios orientados a la prevención secundaria del alcoholismo, los que han tenido altibajos y una suerte muy diversa pero que, con todo, han significado mayores oportunidades para el tratamiento. Respecto de la prevención terciaria, ésta ha estado fundamentalmente en las manos de las organizaciones de alcohólicos recuperados, inicialmente el Club de Abstemios, luego las URACH(Uniones Rehabilitadoras de Alcohólicos) $y$, finalmente, han florecido también grupos de Alcohólicos Anónimos.

En la prevención primaria no ha habido un esfuerzo sistemático, coherente, multiprofesional y multisectorial. Se ha tratado solamente de algunos intentos puntuales no bien elaborados ni implementados, como es el caso del programa nacional de prevención de alcoholismo en el medio escolar, pese a disponerse en el país de profesionales capacitados y con claridad conceptual y experiencia a cerca de lo que debe hacerse en materias de prevención primaria.

Por otra parte, factores como el aumento de la oferta de bebidas alcohólicas, la desorganización social y el incremento de la cesantía, cuya incidencia en el aumento de la prevalencia de la mortalidad por cirrosis hepática alcohólica y del alcoholismo es conocida, hacen temer que la magnitud de los problemas del alcohol y alcoholismo se hayan incrementado o, al menos, neutralizaco los escasos esfuerzos preventivos realizados.

Sin embargo, Pallavicini y col. ${ }^{10}$ afirmam, como resultado de un estudio epidemiológico llevado a cabo en el año 1982 en Santiago, conjuntamente con el censo nacional de población, que hay descenso de la tasa de bebedores excesivos y alcohólicos entre 1958 y 1982, lo que aparece una contradicción con la tasa de mortalidad por

\footnotetext{
* Facultad de Medicina. Universidad de Chile - Casilla 776, Santiago de Chile.
} 
cirrosis hepática en el país, la que habría aumentado en el período (Medina y Kaempffer ${ }^{7}$, Naveillan y Vargas ${ }^{9}$ ). Esta discrepancia puede deberse, en parte o totalmente, a problemas o errores metodológicos en la investigación de Pallavicini y col. ${ }^{10}$. En efecto, Lópes ${ }^{5}$ ha puesto de manifiesto errores muestrales $y$, por otra parte, se empleó simultáneamente dos conceptos y definiciones operacionales del hábito de beber que son incompatibles, lo que hace poco probable que los encuestadores (estudiantes de medicina) las hayan podido manejar con la mínima maestría necesaria para el éxito de la investigación. Además, la validación del instrumento utilizado no fue técnicamente adecuada y se llevó a cabo en enfermos hospitalizados (Pallavicini y col. ${ }^{11}$ ) $y$ en el estudio de campo se utilizó un elevado número de encuestadores (que no se precisa pero que se desprende de la lectura) sin que se analizara los niveles de concordancia entre los mismos ni entre los supervisores del trabajo de terreno (que tambiém fueron varios); no se consideraron las diferencias en la estructura de la población entre 1958 y 1982 al establecer comparaciones con el trabajo de Horwitz ${ }^{4}$, el que se hizo sobre tasas crudas y no ajustadas, que es lo que correspondería para un lapso de 24 años, suficiente para importantes cambios poblacionales; etc.

Medina y col. ${ }^{7}$ muestran un incremento en Chile de las tasas de mortalidad por cirrosis hepática entre los años 1920-24 y 1972 que va de 3,4 a $46,8 \times 100.000$ habitantes, siendo la tasa del período 1955-1959 de 21.9. Se trata de tasas ajustadas a la población chilena total de 1970 . Estas tasas hacen suponer que la prevalencia de alcoholismo ha aumentado en el período, si se analiza ésta de acuerdo con la fórmula de Jellinek*.

Lópes ${ }^{5}$ da las siguientes tasas crudas de mortalidad por cirrosis hepática por 100.000 habitantes (población total): 1950:16,3; 1955:21,67; 1960: 25,$37 ; 1978: 36,17$ y 1982: 29,51. Es decir, las tasas de 1982 serían al menos 4 puntos más altas que las de 1960. Por tratarse de tasas crudas y no ajustadas a la estructura por edades de la población, se hace difícil sacar conclusiones.

Naveillan y Vargas 9 muestran un incremento de las tasas específicas de mortalidad por cirrosis hepática entre 1960 y 1980, tanto en hombres como en mujeres.

Nuestra investigación pretende aportar nuevos elementos de juicio sobre la evolución de las tasas de alcoholismo en las tres décadas que van de 1952 a 1982.

\section{METODOLOGÍA}

La estimación del número de alcohólicos se hizo utilizando la fórmula de Jellinek * que establece:

$$
\text { A ( } N o \text { de alcohólicos) }=\frac{\mathrm{P} \times \mathrm{D} \times \mathrm{R}}{\mathrm{K}}
$$

donde $\mathrm{P}$ es la proporción de defunciones por cirrosis hepática atribuible al alcohol, D son las defunciones por cirrosis hepática, $R$ es la razón de alcohólicos con complicaciones y $\mathrm{K}$ el porcentaje de alcohólicos que mueren por cirrosis hepática alcohólica.

De acuerdo a lo señalado en el Seminario Latinoamericano sobre Alcoholismo ${ }^{13}$, que tuvo lugar en el año 1960, se atribuyen los siguientes valores para la población total (ambos sexos).

$P=37 \%, R=1,6, K=0,694, D=$ defunciones por cirrosis hepática

Elegimos utilizar el $37 \%$ para $\mathrm{P}$ siguiendo la variante II de la aplicación del método de Jellinek ${ }^{13}$, que figura en el señalado documento. Pensamos que finalmente da 10 mismo, para los fines de nuestro estudio, el valor que se dé a $P$ en ausencia de un estudio fidedigno de la mortalidad por cirrosis hepática en hombres y mujeres que nos permita utilizar para cada año un porcentaje exacto; otro tanto es válido para $\mathrm{K}$ y $\mathrm{R}$. En efecto, esto nos va a permitir establecer una base común de medida para los diferentes años lo que posibilitará establecer comparaciones de validez aceptable, independientemente de que para cada año se subestime o sobreestime al número real de alcohólicos, si el sesgo es el mismo para todos los años.

El problema con esta presunción radica en que los valores de $P, R$ y $K$ pueden ser distintos para los distintos años, pero no tenemos como resolver este problema. Este punto se tendrá en cuenta en la discusión de los resultados.

Otro aspecto metodológico a retener es que el concepto de alcoholismo que Jellinek* utilizó al establecer esta fórmula corresponde, en cierto modo, a lo que nosotros llamamos bebedores problemas, concepto que abarca a los bebedores excesivos y alcohólicos propiamente tales (ver Naveillan ${ }^{8}$ ), pero incluyendo fundamentalmente a todos los últimos y a pocos de los primeiros. En el texto ocupamos el término alcohólico en este sentido más amplio (salvo que especifiquemos otra cosa) que le da Jellinek ${ }^{13}$, ya que su método no nos permite separar ambos grupos.

\section{RESULTADOS}

En la Tabla 1 tenemos el número estimado 
TABLA 1

Numero y tasa de alcoholicos según sexo* (poblacion de 15 y mas años), Chile, $1952-1982$

\begin{tabular}{|c|c|c|c|c|c|c|}
\hline \multirow[b]{2}{*}{ Año } & \multicolumn{2}{|c|}{ Hombres } & \multicolumn{2}{|c|}{ Mujeres } & \multicolumn{2}{|c|}{ Total } \\
\hline & NAlcohólicos & Tasa $\times 100$ & $N^{*}$ Alcohólicos & Tasa $\times 100$ & $N^{2}$ Alcohólicos & Tasa $\times 100$ \\
\hline 1952 & 55.020 & 3,0 & 25.847 & 1,3 & 80.86 & 2,1 \\
\hline 1953 & 47.684 & 25 & 21.923 & 1,1 & 69.607 & 1,7 \\
\hline 1954 & 63.721 & 3,2 & 32.756 & 1,5 & 96.477 & 2,3 \\
\hline 1955 & & - & & - & & - \\
\hline 1956 & 98.524 & 4,6 & 40.945 & 1,8 & 139.469 & 3,2 \\
\hline 1957 & 78.137 & 3,6 & 38.386 & 1,7 & 116.523 & 26 \\
\hline 1958 & 87.094 & 3,9 & 40.604 & 1,7 & 127.698 & 2,8 \\
\hline 1959 & 105.605 & 4,7 & 52.375 & 2,2 & 157.980 & 3,4 \\
\hline 1960 & 115.670 & 5,2 & 52.034 & 2,2 & 167.704 & 3,6 \\
\hline 1961 & 118.656 & 5,2 & 46.034 & 1,9 & 165.061 & 3,5 \\
\hline 1962 & $\ldots$ & - & $\ldots$ & - & $\ldots$ & - \\
\hline 1963 & 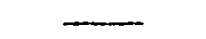 & - & 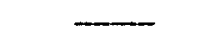 & - & + & - \\
\hline 1964 & 192.869 & 7,9 & 72.934 & 2,8 & 265.803 & 52 \\
\hline 1965 & 158.578 & 6,4 & 55.617 & 2,1 & 214.195 & 4,1 \\
\hline 1966 & 174.188 & 6,8 & 74.640 & 2,7 & 248.828 & 4,7 \\
\hline 1967 & 201.399 & 7,6 & 83.170 & 2,9 & 284.569 & 5,2 \\
\hline 1968 & 208.130 & 7,6 & 93.577 & 3,2 & 301.715 & 5,3 \\
\hline 1969 & 202.594 & 7,2 & 84.450 & 2,8 & 287.044 & 5,0 \\
\hline 1970 & - & - & $\underline{L}$ & - & 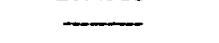 & - \\
\hline 1971 & 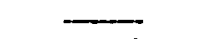 & - & - & - & - & - \\
\hline 1972 & 290.455 & 9,7 & 107.761 & 3,2 & 399.216 & 6,5 \\
\hline 1973 & 204.129 & 6,7 & 72.848 & 23 & 276.977 & 4,4 \\
\hline 1974 & 169.667 & $5 / 4$ & 67.901 & 2,1 & 237.568 & 3,7 \\
\hline 1975 & 166.084 & 5,2 & 64.659 & 1,9 & 230.743 & 3,5 \\
\hline 1976 & 169.411 & 5,1 & 67.389 & 1,9 & 236.800 & 3,5 \\
\hline 1977 & 192.357 & 5,7 & 76.090 & 2,1 & 268.447 & 3,9 \\
\hline 1978 & 234.411 & 6,7 & 90.080 & 2,5 & 324.491 & 4,5 \\
\hline 1979 & 218.375 & 6,1 & 92.895 & 25 & 311.270 & 43 \\
\hline 1980 & 198.329 & 5,4 & 79.673 & 21 & 276.002 & 3,7 \\
\hline 1981 & 201.144 & 5,4 & 76.346 & 2,0 & 277.490 & 3,6 \\
\hline 1982 & 208.991 & 5,5 & 77.711 & 1,95 & 286.702 & 3,66 \\
\hline
\end{tabular}

Tasa no ajustada por estructura etárea de la problación

de alcohólicos a partir de las muertes por cirrosis hepática, calculado de acuerdo a la fórmula de Jellinek ${ }^{13}$, y la tasa de alcohólicos para la población chilena de 15 años y más, según sexo y total, de los años que van de 1951 a 1982, con la excepción de 1955, 1962, 1963, 1970 y 1971, para los cuales no dispusimos de la información necesaria.

Como se trata de un período de algo más de 30 años y la estructura de la población, que ha crecido, puede haberse modificado sustancialmente, hemos procedido a ajustar los resultados a la población chilena de 1984 con la finalidad de establecer comparaciones de mayor validez. Este ajuste se presenta en la Tabla 2 respecto de los años que elegimos para estes fines y que son 1952, $1956,1958,1960,1964,1972$ y 1982. Si comparamos las tasas ajustadas de acuerdo a la estructura etárea de población de estos años con las no ajustadas que aparecen en la Tabla 1, nos encontramos que son muy similares.
Estos resultados nos muestran que el número total de alcohólicos ha aumentado de 80.867 en 1952, a 286.702 en 1982 y que la tasa de ellos lo ha hecho de $2,1 \%$ a $3,66 \%$. Si tomamos los años 1958 y 1982, para los cuáles existen estudios epidemiológicos de campo en Santiago, el incremento va de 127.698 (tasa $2,8 \%$ ) a 286.702 (tasa 3,66\%) (Tabla 1).

Si hacemos las comparaciones en base a tasas ajustadas a la estructura de población (Tabla 2), tenemos que el incremento entre 1952 y 1982 va de 178.112 alcohólicos (tasa 2,19\%) a 301.459 (tasa $3,7 \%$ ) lo que representa un $69,3 \%$ de aumento en el número y de $69,9 \%$ en la tasa.

El aumento de la tasa ajustada y del número de alcohólicos entre los años 1958 y 1982 es de $28,9 \%$.

En síntesis, de estas dos tablas se desprende que para 1982, respecto de 1952 y de 1958 (y de 
TABLA 2

Tasas de alcoholismo estimadas a partir de tasas ajustadas de mortalidad por cirrosis hepatica. (Poblacion de 15 y mas años), Chile, 1952 - 1982

\begin{tabular}{|c|c|c|c|c|c|}
\hline \multirow{2}{*}{$\begin{array}{c}\begin{array}{c}\text { Tasas } \\
\text { Año }\end{array} \\
1952\end{array}$} & \multirow{2}{*}{$\begin{array}{c}\begin{array}{c}\text { Población } \\
\text { Base }\end{array} \\
1984\end{array}$} & \multicolumn{2}{|c|}{$\begin{array}{c}\text { Defunciones } \\
\text { Esperadas } \\
\text { por Cirrosis }\end{array}$} & \multirow{2}{*}{$\begin{array}{c}\begin{array}{c}N^{2} \text { esperado* } \\
\text { de Alcohólicos } \\
\text { (Mét. de Jellinek) }\end{array} \\
\begin{array}{c}178.112 \\
120.703 \\
57.409\end{array}\end{array}$} & \multirow{2}{*}{$\begin{array}{c}\begin{array}{c}\text { Tasa Esperada } \\
\text { de Alcoholismo } \\
\%\end{array} \\
2,19 \\
3,03 \\
1,38\end{array}$} \\
\hline & & $\begin{array}{l}\text { Total } \\
\text { Hombres } \\
\text { Mujeres }\end{array}$ & $\begin{array}{r}2.088 \\
1.415 \\
673\end{array}$ & & \\
\hline 1956 & 1984 & $\begin{array}{l}\text { Total } \\
\text { Hombres } \\
\text { Mujeres }\end{array}$ & $\begin{array}{r}3.143 \\
2.185 \\
958\end{array}$ & $\begin{array}{r}268.106 \\
186.386 \\
81.720\end{array}$ & $\begin{array}{l}3,29 \\
4,67 \\
1,97\end{array}$ \\
\hline 1958 & 1984 & $\begin{array}{l}\text { Total } \\
\text { Hombres } \\
\text { Mujeres }\end{array}$ & $\begin{array}{r}2.742 \\
1.844 \\
898\end{array}$ & $\begin{array}{r}233.900 \\
157.298 \\
76.602\end{array}$ & $\begin{array}{l}2,87 \\
3,94 \\
1,84\end{array}$ \\
\hline 1960 & 1984 & $\begin{array}{l}\text { Total } \\
\text { Hombres } \\
\text { Mujeres }\end{array}$ & $\begin{array}{l}3.402 \\
2.330 \\
1.072\end{array}$ & $\begin{array}{r}290.199 \\
198.755 \\
91.444\end{array}$ & $\begin{array}{l}3,56 \\
4,98 \\
2,20\end{array}$ \\
\hline 1964 & 1984 & $\begin{array}{l}\text { Total } \\
\text { Hombres } \\
\text { Mujeres }\end{array}$ & $\begin{array}{l}5.009 \\
3.632 \\
1.377\end{array}$ & $\begin{array}{l}427.281 \\
309.819 \\
117.462\end{array}$ & $\begin{array}{l}5,24 \\
7,77 \\
2,82\end{array}$ \\
\hline 1972 & 1984 & $\begin{array}{l}\text { Total } \\
\text { Hombres } \\
\text { Mujeres }\end{array}$ & $\begin{array}{l}6.260 \\
4.557 \\
1.703\end{array}$ & $\begin{array}{l}533.994 \\
388.724 \\
145.270\end{array}$ & $\begin{array}{l}6,55 \\
9,75 \\
3,49\end{array}$ \\
\hline 1982 & 1984 & $\begin{array}{l}\text { Total } \\
\text { Hombres } \\
\text { Mujeres }\end{array}$ & $\begin{array}{r}3.534 \\
2.577 \\
957\end{array}$ & $\begin{array}{r}301.459 \\
219.825 \\
81.635\end{array}$ & $\begin{array}{l}3,70 \\
5,51 \\
1,96\end{array}$ \\
\hline
\end{tabular}

Estimaciones según métodos de Jellinek

otros años), ha habido no sólo un aumento del número de alcohólicos que no se explica sólo por el crecimiento demográfico del período, en base a una tasa de alcoholismo constante, sino que también un incremento llamativo de las tasas de prevalencia (según el método de Jellinek ${ }^{13}$ ) y, por ende, de incidencia del alcoholismo. Esto se pone de manifiesto con los ajustes de población.

Sin embargo, el aumento no ha sido uniforme sino que discontinuo en el tiempo, como se desprende del análisis de las Figuras 1 y 2.

En la Figura 1 tenemos la variación del número de alcohólicos en el período que va de 1952 a 1982: en la línea con cuadros blancos de acuerdo a las defunciones brutas registradas cada año y en la con cuadros negros, de acuerdo a las estimadas para 1984 a partir de las registradas en los años 1952, 1956, 1958, 1960, 1964, 1972 y 1982, según procedimiento de ajuste poblacional.

Se aprecia un incremento discontinuo en la línea de los números brutos de alcohólicos, con un máximo para 1972 y algunas caídas bruscas para los años 1965, 1975 y 1980, todas las cuales se mantienen muy por encima del nivel de 1952 . En la curva de los números estimados se tiene el mismo incremento, resultando, como es obvio por el ajuste poblacional, un mayor número de alcohólicos por ser mayor la población que ha servido de referencia.

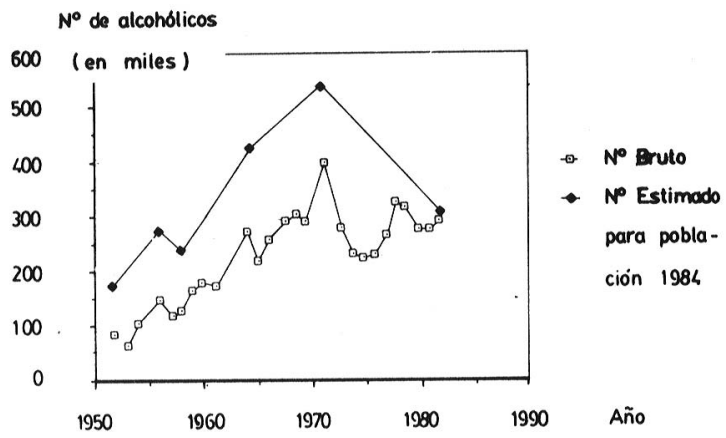

Fig. 1 - Variación en numero de alcohólicos, Chile, 1952-1982

En la Figura 2 se presentan las tasas de alcoholismo respectivas, sin ajuste poblacional. Fue- 
ra de las oscilaciones sefaladas, se aprecia que los niveles de 1982 son superiores, al menos, a los de 1952 y 1958.

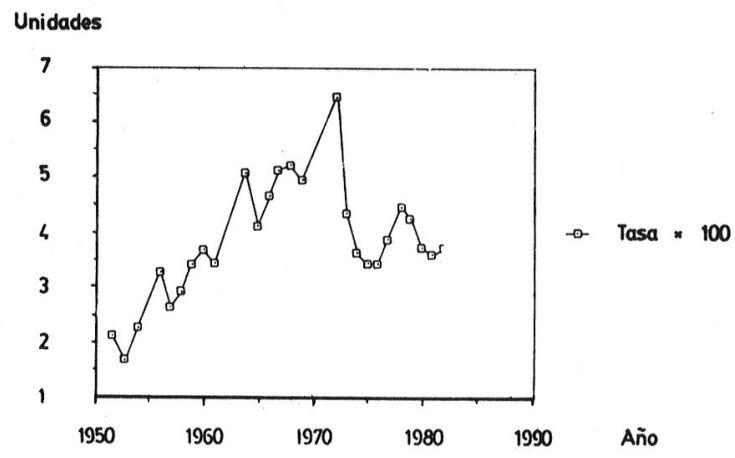

Fig. 2 - Variaciones en la tasa de alcoholicos (sin ajuste poblacional), Chile, 1952-1980.

En la Figura 3 se presenta las tasas de alcoholismo de los años del perído estudiado, ajustadas para la población de 1984 y según sexo. Se aprecia que la tasa de alcoholismo es mayor en los hombres que en las mujeres.

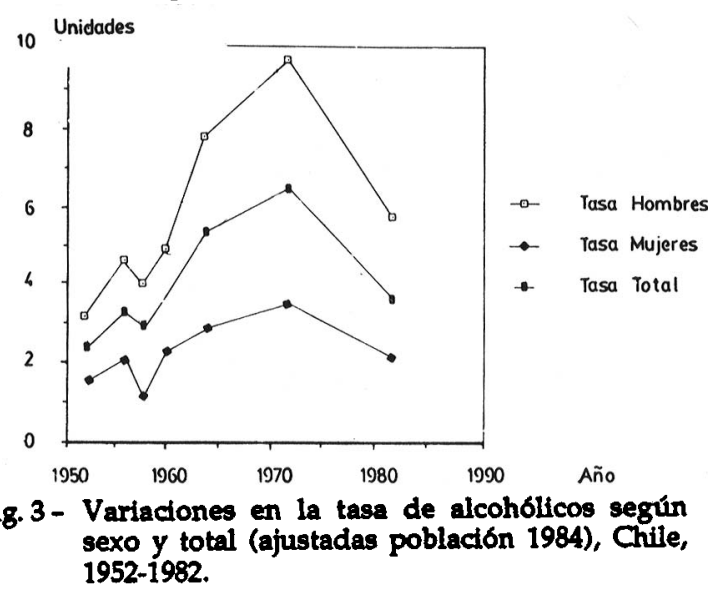

Del análisis de las tres figuras presentadas se desprende que la década que va del año 1964 al 74 fue el peor periodo en cuanto a número total de alcohólicos, tasas de alcoholismo y de mortalidad por cirrosis hepática (de la cual se derivan los otros datos); hubo un nuevo repunte en el año 1978 y pareciera ser que se insinúa otro a contar de 1980 (Figs. 1 y 2).

Llama la atención la gran caída del número y tasa de alcohólicos a partir de 1972, que fue su nivel más alto. Una explicación parcial de esto es que el incremento de las muertes por cirrosis hepática hacia 1972 deja pocos cirróticos que puedan morirse en los años siguientes. Seguramente juegan también un papel importante factores relativos a la oferta, precio y consumo de bebidas alcohólicas y variables relacionadas con la organización/desorganización social, entre otras. La dilucidación de este fenómeno requiere de otros estudios.

\section{DISCUSIÓN}

El presente estudio tiene limitaciones que deseamos señalar.

En primer lugar están todas las relativas al método mismo, o fórmula de Jellinek ${ }^{13}$, para estimar el número de alcohólicos, fórmula que, con todo, ha dado en varios países resultados bastante próximos de los obtenidos en encuestas de campo. En algunos países latinoamericanos, como Chile y Perú, los resultados han sido bastante disímiles, lo que es debido, en parte, a diferencias en la metodología de los estudios de campo y en parte a que el valor de los constantes $P, R$ y $\mathrm{K}$ de la fórmula han sido establecidas arbitrariamente para nuestros países a falta de mejor información. Además, las constantes $\mathrm{K}$ ( $\%$ de todos los alcohólicos con complicaciones que mueren de cirrosis hepática alcohólica) y $\mathrm{R}$ (razón de los alcoholicos con complicaciones) pueden haber cambiado con el correr de los años por factores nutricionales y de mejor calidad en la atención médica, por ejemplo. Otro tanto puede ocurrir con $\mathrm{P}$ (proporción de defunciones por cirrosis hepática que pueden ser atribuidas al alcohol) con el agravante de que en nuestro medio seguramente esta proporción es mayor que la encontrada en Estados Unidos(62,8\%) y, además, utilizamos el mismo valor de $37 \%$ propuesto para la sumatoria de ambos sexos al considerarlos separadamente lo que puede traducirse en una subestimación de los alcohólicos hombres y en una sobre-estimación de los alcoholicos mujeres y, por lo tanto, en una disminución de la relación hombre/mujer alcoholicos.

Pensamos que las limitaciones que nacen de los valores $\mathrm{K}$ y $\mathrm{R}$ podrían tener mayor peso té́rico que no práctico por cuanto el tratamiento de la cirrosis hepática no ha mejorado sensiblemente su pronóstico y los factores nutricionales de la población mayor de 15 años no parecen haber cambiado espectacularmente en un sentido positivo.

En cuanto a $P$, es posible que haya aumentado la proporción de cirrosis hepática alcoholica entre mujeres que padecen de cirrosis hepática pero en los hombres lo más probable es que se haya mantenido igual.

Para los fines prácticos de esta investigación, y a falta de mejor información, nos parece que el mantener un factor constante a través del tiempo para la conversión de muertos por cirrosis hepática a alcohólicos, se justifica y nos permite establecer comparaciones con razonable validez pese a que se subestime o sobreestime al por 
igual el número de alcohólicos en todos los años de las tres décadas contempladas.

El segundo grupo de limitaciones nace del valor de las estadísticas de defunciones utilizadas, que son las oficiales de Chile, y de si los errores que pudieran contener han sido o nó los mismos durante este largo período de tiempo. Es posible que la información estadística haya mejorado al correr de los años disminuyendo los subregistros y que esto pueda, en parte, explicar las diferencias encontradas. Sin embargo, dada la tradición existente en nuestros registros vitales, no creemos que sea así. Medina ${ }^{7}$ es de similar parecer.

Un tercer sesgo puede originarse en las estimaciones de población, siempre oficiales, para los años en que no ha habido censo. Pensamos que esta posibilidad de error se encuentra suficientemente controlada por la Dirección de Estadística y Censo.

Vistas las cosas así, el número de defunciones por cirrosis hepática y el número de alcohólicos estimado de acuerdo a la fórmula de Jellinek, se encuentran necesariamente en estricto paralelismo y las conclusiones que se sacan para uno son válidas para el otro. Las tasas de alcoholismo resultantes están referidas a la población, como corresponde, y guardan, por tanto, relación con la magnitud de ésta y el número de alcohólicos calculados en base a la fórmula de Jellinek ${ }^{13}$; la influencia de la estructura de la población en ellas se controló al ajustarlas para la población de 1984, como se senala en resultados.

Entrando de lleno a la discusión de nuestros resultados, llaman la atención las bruscas alzas y caídas del número de alcohólicos y de las tasas respectivas, particularmente las "crestas" de 1964 y 1972. Pensamos que un factor de explicación de estas súbitas variaciones está en factores externos como la oferta y el precio relativo de las bebidas alcohólicas. Brenner ${ }^{1}$, al igual de Terris ${ }^{14}$, señala que el consumo del alcohol está directamente relacionado, a corto plazo, con la mortalidad por cirrosis hepática, con un tiempo de precedencia de entre 1 a 2 años del primero respecto de la segunda. Si esto es así, un aumento del consumo explicaría el aumento de la mortalidad por cirrosis hepática (y del número y tasa de alcohólicos) en un año. En estas condiciones morirían antes aquellos que en condiciones estables de consumo habrían tardado más años en hacerlo. El brusco descenso que sigue a los años de máxima reflejaría estas muertes más precoce a más de otros factores externos como la disminución de la oferta de bebidas alcohólicas. Es posible, sin embargo, que esta explicación no sea suficiente.
Otro aspecto a retener es la tendencia al alza del número y tasa de alcohólicos entre 1952 y 1972 para luego caer bruscamente ese año y a continuación remontar hasta 1978 , descendiendo luego para estabilizarse o iniciar un repunte en 1980 (Figs 1 y 2). Estas variaciones a largo plazo de estas tasas y de número de alcohólicos podrían explicarse por factores socio económicos, como lo señala Brenner 1, que habría que investigar.

Hemos encontrado que, en las 3 décadas transcurridas entre 1952 y 1982, las tasas y número de alcohólicos se han incrementado en cerca de un $70 \%$ lo que implica, de ser ciertos nuestros hallazgos, la inexistencia de una adecuada política de prevención del alcoholismo o su ineficacia o fracaso.

Como nuestros hallazgos se encuentran en contradicción con las conclusiones a que llegaron Pallavicini y col. ${ }^{10}$ al comparar las tasas de alcoholismo del gran Santiago correspondientes a los años 1958 y 1982, nos hemos centrado en ellos y hemos encontrado que para el país ha habido un incremento del orden del $30 \%$ entre estos años. Si bien en la introducción hemos señalado las limitaciones metodológicas de las investigaciones de dichos autores, debemos analizar algo más esta discrepancia.

Mirando solamente los resultados del trabajo de Pallavicini y col. ${ }^{10}$ y estableciendo los porcentajes del caso, tenemos que para ellos los alcohólicos han aumentado en un $17,6 \%$ los bebedores excesivos han disminuido en un $30,8 \%$. La formula de Jellinek incluye necesariamente en su concepto de alcoholismo a bebedores excesivos ya que muchos de estos también fallecen por cirrosis hepática alcohólica. Es por esta última razón que nuestros reslultados son aún más contradictorios con los de dichos autores.

Es posible que una parte de la diferencia sea explicable, aunque no lo cremos así, por el hecho de que los resultados de Pallavicini y col. ${ }^{10}$ están referidos al gran Santiago y los nuestros a todo Chile.

Queremos señalar, finalmente, que nuestras cifras y resultados respecto de los diferentes años valen por sí mismos ya que hemos trabajando com la población total y no con muestras, es decir, no hay variación muestral.

\section{CONCLUSIONES}

1. Ha habido un incremento en Chile del número y de la tasa de alcohólicos entre los años 1952 y 1982 . Esta alza es del orden del $70 \%$ para ambos indicadores. 
2. Si se toma como base el año 1958 , este incremento ha sido del $30 \%$.

3. Este incremento no ha sido continuo sino que irregular, registrándose un máximo de alcohólicos en el año 1972 para luego disminuir hacia 1980, fecha en que se insinúa un incremen- to. Con las tasas ocurre algo similar pero con estabilización después de 1980.

4. Nuestros resultados apuntan a la inexistencia o a la ineficacia de las políticas de prevención del alcoholismo en el período contemplado en el estudio.

NAVEILLAN, P. \& VARGAS, S. Prevalência do alcolismo durante três décadas no Chile (1952-1982), Rev. Saúde públ., S. Paulo, 23:128-35, 1989.

RESUMO: Mediante o método de Jellinek foi estudada a evolução do número de alcóolatras e da taxa de prevalência do alcoolismo no Chile e suas variaçōes entre os anos de 1952 e 1982 . Foram assinalados os viéses e limitações da metodologia empregada e comparados os resultados obtidos com os de outros estudos chilenos. Concluiu-se que houve aumento do número de alcoblatras e da taxa de alcoolísmo, no período analisado, sendo que a taxa observada em 1982 foi $70 \%$ maior que a de 1952. Este aumento mostrou-se irregular e flutuante. Os resultados questionam a efícácia das polfticas de prevençăo do alccolismo, realizadas no período.

DESCRITORES: Alcoolismo, incidência. Prevalência.

NAVEILLAN, P. \& VARGAS, S. [The prevalence of alcoholism in chile over three decades (19521982)]. Rev. Saúde públ., S. Paulo, 23:128-35, 1989.

ABSTRACT: The level of alcoholism in Chile and its variations between 1952 and 1982 are studied by means of the Jellinek Method. The limitations and bias of the methodology employed are indicated and the results are compared with those of other children studies. The conclusion is that there was an increase in the number of alcoholics and in the alcoholism rate during the period analyzed, with a rate for $198270 \%$ higher than that for 1952 . The efficacy of the policy for the prevention of alcoholism adopted in the period under study is called in question by the results presented.

KEYWORDS: Alcoholism, occurrence. Prevalence.

\section{REFERÊNCIAS BIBLIOGRÁFICAS}

1. BRENNER, M. H. Trends in alcohol consumption and associated illnesses: some effects of economic changes. Amer. J.publ. Hlth, 65: 1279-92, 1975.

2. HONORATO, R. et al .Prevalencia de alcoholismo en la $5^{3}$ Circunscripción de Santiago. Apud Simposium sobre Alcoholismo y Problemas Derivados del Alcohol, Santiago, Chile, Servicio Nacional de Salud, 1957.

3. HORWTTZ, J. \& HONORATO, R. Importancia del alcoholismo y de los problemas derivados del alcohol. Rev. Serv. nac. Salud, 2: 243-57, 1957. [Simposium sobre Alcoholismo y Problemas Derivados del Alcohol, Santiago de Chile, 1957].

4. HORWITZ, J. \& MUNOZ, L.C. Investigaciones epidemiológicas a cerca de la mortalidad mental en Chile. Rev. Serv. nac. Salud, 3: 277-309, 1958.

5. LOPEZ, A. Aspectos economicos del alcoholismo. $l^{\text {a }}$ parte: el costo del alcoholismo en Chile, 1981. Santiago, Chile, Departamento de Economía de la Universidad de Chile, 1984.
6. MARCONI, J, et al. A survey of the prevalence of alcoholism among the adult population of a suburb of Santiago. Quart. J. Stud. Alcohol., 16: 438 - 46, 1955.

7. MEDINA, E. \& KAEMPFFER, A. Epidemiología de la cirrosis hepática en Chile. Rev. méd. Chile, 102: 466, 1974.

8. NAVEILLAN F., P. Sobre el concepto de alcoholismo. Bol. Ofic. sanit. ponamer., 91: 340-8, 1981.

9. NAVEILLAN F., P. \& VARGAS, S. Expectativa de vida del bebedor problema en Santiago de Chile. V - Proyección de resultados de 1960 a 1980. Bol. Ofic. Sanit. panamer., 97: 150-8, 1984.

10. PALLAVICINI, J, et al. Estudio comparativo sobre alcoholismo y otros hábitos de beber en Santiago, 19581982. Rev. Psiq. clin., 20: 48-67, 1983.

11. PALLAVICINI, J. et al. Validación de un instrumento para el diagnóstico de alcoholismo en la población. Rev. Psiq. clin., 20: 36-41,1983. 
12. ROSENBLATT, E. et al. Estudio de algunas condiciones socio- económicas en relación con el alcoholismo en una población de Santiago. Int. J. Alcohol., 1: 163-70,1956.

13. SEMINARIO Latinoamericano sobre Alcoholismo. Viña del Mar, Chile, Organización Panamericana de la Salud, 1960.
14. TERRIS, M. Epidemiology of cirrosis of the liver: national mortality data. Amer. J. publ. Hlth, 57: 2076-88, 1967.

Recebido para publicaçăo em 9/9/1988 Aprovado para publicaçäo em 22/12/1988 